SAÚDEE AMBIENTE

V.8・N.1・Agosto/Setembro/Outubro - 2019

ISSN Digital: 2316-3798

ISSN Impresso: 2316-3313

DOI: $10.17564 / 2316-3798.2019 v 8 n 1 p 81-98$

ORCID: 0000000000000
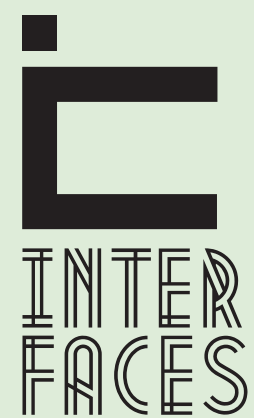

CIENTÍFICAS

\section{ATIVIDADE FÍSICA E CAPACIDADE FUNCIONAL PARA AS ATIVIDADES DA VIDA DIÁRIA DE IDOSOS USUÁRIOS DE UNIDADES BÁSICAS DE SAÚDE}

PHYSICAL ACTIVITY AND FUNCTIONAL CAPACITY FOR DAILY LIFE ACTIVITIES OF ELDERLY USERS OF BASIC HEALTHUNITS

ACTIVIDAD FÍSICA Y HABILIDAD FUNCIONAL PARA ACTIVIDADES

DE VIDA DIARIA DE USUARIOS MAYORES DE UNIDADES DE SALUDBÁSICAS

\section{RESUMO}

Esse presente estudo teve como objetivo analisar o nível de atividade física e a capacidade funcional dos idosos usuários das Unidades Básicas de Saúde (UBS) de um município do noroeste paranaense. Estudo transversal, realizado com 654 idosos, de ambos os sexos. Foram utilizados o International Physical Activity Questionnaire, a Escala de Katz e a Escala de Lawton. A análise dos dados foi realizada por meio dos testes Kolmogorov-Smirnov. Qui-quadrado, Kruskal-Wallis, "U” de Mann-Whitney e Spearman. Considerou-se um nível de significância de $p<0,05$. Os idosos com idade entre 60 e 69 anos e que procuram as UBS para consulta médica apresentaram melhor capacidade funcional nas atividades básicas de vida diária (ABVD) e nas atividades instrumentais de vida diária (AIVD). Os idosos não aposentados e com maior nível de atividade física apresentaram melhor capacidade funcional nas AIVD. Idosos sem histórico de quedas demonstraram melhor capacidade funcional nas ABVD. Existe tendência dos idosos com boa percepção de saúde e independência nas ABVD e AIVD terem nível de atividade física muito ativo/ ativo. Variáveis sociodemográficas e condições de saúde estão associadas ao nível de atividade física e à capacidade funcional de idosos.

\section{PALAVRAS-CHAVE}

Nathan Faleiro Rodrigues ${ }^{2}$ Celita Salmaso Trelha ${ }^{3}$ José Roberto Andrade do Nascimento Júnior ${ }^{4}$

Daniel de Aguiar Pereira ${ }^{5}$

Mateus Dias Antunes ${ }^{6}$

Sônia Maria Marques Gomes Bertolini ${ }^{7}$
Atividade motora, Exercício, Gerontologia, Promoção da saúde. 


\section{ABSTRACT}

This study aimed to analyze the level of physical activity and functional capacity of the elderly users of the Basic Health Units (BHU) of a municipality in the northwest of Paraná. A cross-sectional study was carried out with 654 elderly people of both sexes. The International Physical Activity Questionnaire, the Katz Scale and the Lawton Scale were used. Data analysis was performed using the Kolmogorov-Smirnov tests. Qui-square, Kruskal-Wallis, Mann-Whitney's “ $U$ ” test and Spearman. A significance level of $p<0.05$ was considered. The elderly with ages ranging from 60 and 69 years of age and who seek the UBS for medical consultation had better functional capacity in the basic activities of daily living (ABVD) and instrumental activities of daily living (AIVD). The elderly, who were not retired and had a higher level of physical activity, had better functional capacity in the AIVD. Elderly patients with no history of falls demonstrated better functional capacity in ABVD. There is a tendency of the elderly with good perception of health and independence in ABVD and AIVD to have very active / active level of physical activity. Sociodemographic variables and health conditions are associated with the level of physical activity and functional capacity of the elderly.

\section{KEYWORDS}

Motor activity, Exercise, Gerontology, Health promotion.

\section{RESUMEN}

El objetivo de este estudio fue analizar el nivel de actividad física y la capacidad funcional de los usuarios ancianos de las Unidades Básicas de Salud (BHU) de una ciudad en el noroeste de Paraná. Estudio transversal con 654 hombres y mujeres mayores. Se utilizaron el cuestionario internacional de actividad física, la escala de Katz y la escala de Lawton. El análisis de los datos se realizó utilizando las pruebas de Kolmogorov-Smirnov, Chi-cuadrado, Kruskal-Wallis, "U” de Mann-Whitney y Spearman. Se consideró un nivel de significación de $p<0.05$. Las personas mayores de 60 e 69 años y que buscan el BHU para consulta médica mostraron una mejor capacidad funcional en las actividades básicas de la vida diaria (BADL) y en las actividades instrumentales de la vida diaria (IADL). Los adultos mayores no jubilados y con mayor nivel de actividad física presentaron una mejor capacidad funcional en el IADL. Las personas mayores sin antecedentes de caídas demostraron una mejor capacidad funcional en ABVD. Hay una tendencia de los ancianos con buena percepción de salud e independencia en BADL y IADL a tener un nivel muy activo / activo de actividad física. Las variables sociodemográficas y las condiciones de salud están asociadas con el nivel de actividad física y la capacidad funcional de los ancianos. 


\section{PALABRAS CLAVE}

Actividad motora, Ejercicio, Gerontología, Promoción de la salud.

\section{INTRODUÇ̃̃o}

O envelhecimento populacional é um fenômeno mundial decorrente de mudanças culturais e melhorias nas condições de vida (ALVES et al., 2014). Porém, o aumento da expectativa de vida só vale à pena se for vivido com uma boa qualidade de vida (LOPES; SANTOS, 2015).

O sedentarismo é tido com um dos principais fatores de risco aos agravos à saúde e um dos mais prevalentes na população mundial e brasileira; sendo atualmente considerado como um grave problema de saúde pública (SIQUEIRA et al., 2009). No Brasil, a inatividade física aumentou em pessoas com baixa renda entre os anos de 2002 e 2007 e a prevalência de inatividade física aumentou de 41,1\% em 2002 para 52,0\% em 2007 (KNUTH et al., 2010). Outros dados mostram que a inatividade física aumenta com a idade e que pessoas acima de 60 anos são as mais acometidas (HALLAL et al., 2012). Devido a esses altos níveis de inatividade física no Brasil, em 2010 foi criada, junto a rede básica de saúde, a Política Nacional de Promoção de Saúde, a qual tem em um dos seus eixos a prática de atividade física. Com isso, programas de promoção de atividade física foram criados e financiados pelo governo brasileiro com objetivo de diminuir o sedentarismo e melhorar a qualidade de vida (MALTA et al., 2014).

Dentre os aspectos dessa qualidade, a funcionalidade preservada é apontada pelos idosos como um dos mais importantes mediadores da independência e autonomia (LOPES; SANTOS, 2015). A Classificação Internacional de Funcionalidade, Incapacidade e Saúde (CIF) utiliza como termo a funcionalidade, que engloba as funções corporais, atividade e a participação social. Por outro lado, a incapacidade é utilizada no aspecto negativo e abrangem situações nas quais se manifestam deficiências, limitações e restrições à participação em atividades. Portanto a CIF tem como alicerce a abordagem biopsicossocial, que incorpora os componentes de saúde nos níveis corporais e sociais, fornecendo subsídios para políticas de inclusão social (FARIAS; BUCHALLA, 2005).

A capacidade funcional é hierarquizada em três níveis - básica, instrumental e avançada. As atividades básicas de vida diária (ABVD) (KATZ et al., 1963) incluem domínios relacionados ao autocuidado e a sobrevivência (como tomar banho, alimentar-se, transferir-se, vestir-se, e usar a toalete), as atividades instrumentais da vida diária (AIVD) (LAWTON et al., 1969) envolvem tarefas mais adaptativas para vida na comunidade (cuidar da casa, preparar as refeições, lavar roupa, usar transporte, fazer compras, usar o telefone, controlar o dinheiro e os medicamentos) e podem ser avaliadas separadamente ou em conjunto (KATZ et al., 1963; LAWTON et al., 1969). As atividades avançadas de vida diária (AAVD) colocam os idosos em contato com papéis e com a vida social, ou seja, se enquadram no complexo conjunto de competências comportamentais associadas à funcionalidade, à motivação 
e às experiências anteriores, exigindo autonomia cognitiva, bom nível de independência física e consequentemente AIVD e ABVD preservadas (SPOSITO et al., 2016).

0 envelhecimento acarreta mudanças fisiológicas e neuromusculares relacionadas à diminuição (em números) das fibras musculares de contração rápida (tipo II) e, consequentemente, a redução da área transversal de cada fibra muscular (BERTOLI et al., 2017). Essa redução predispõe a sarcopenia, termo usado para definir a perda de massa e força muscular relacionada à idade (CRUZ-JENTOFT, 2010). Como resultado, os idosos se tornam mais lentos para a realização das atividades de vida diária (AVD) progredindo para o declínio funcional manifestado pela vulnerabilidade (PAULA et al., 2015).

Diante das alterações neuromusculares decorrentes do processo de envelhecimento, a atividade física aparece como um importante mediador para retardar os declínios funcionais e reduzir o aparecimento de doenças crônicas em idosos saudáveis e, consequentemente, preservando a autonomia e independência das AVD (CORDEIRO et al., 2014; JACINTO et al., 2017). Considerando o papel da rede de Atenção Básica à Saúde, o presente estudo teve como objetivo analisar o nível de atividade física e a capacidade funcional dos idosos usuários das UBS de um município do noroeste paranaense.

\section{MÉTODO}

\section{Participantes}

O processo de amostragem deste estudo epidemiológico de corte transversal foi realizado em dois estágios. Inicialmente foram elencadas as UBS das quatro regiões da cidade: Leste (7 UBS) que contempla $21,8 \%$ da população, região norte (8 UBS) com $34,5 \%$ da população, região oeste (8 UBS) com 23,2\% da população e a região Sul (8 UBS) que contempla 20,4\% da população total de idosos. A partir disso, foram selecionadas de forma aleatória três UBS de cada região geográfica para participar da pesquisa, totalizando 12 UBS. Conforme dados da Secretária de Saúde de Maringá-PR de 2016, aproximadamente 42.000 idosos foram atendidos pelas UBS do município. A partir dessa população-alvo, a amostra a ser considerada foi de 654 idosos considerando possíveis perdas amostrais, os quais foram selecionados por conveniência. Adotou-se nível de confiança de $95 \%$ e $4 \%$ de margem de erro e o software utilizado para obtenção dos cálculos foi o StatDisk versão 8.4 .

Foram incluídos no estudo idosos de ambos os sexos com idade igual ou superior a 60 anos, com capacidade de fala e audição preservadas, que permitiam a aplicação dos questionários. Foram excluídos idosos com possíveis déficits cognitivos, avaliados pelo Mini Exame do Estado Mental (MEEM) (FOLSTEIN et al., 1983). As notas de corte utilizadas para exclusão pelo MEEM foram: 17 para os analfabetos; 22 para idosos com escolaridade entre 1 e 4 anos; 24 para os com escolaridade entre 5 e 8 anos e 26 os que tiverem 9 anos ou mais anos de escolaridade. Correspondem à média obtida por esses autores para cada faixa de escolaridade, menos um desvio padrão (BRUCKI et al., 2003). Idosos classificados abaixo do ponto de corte específico para sua escolaridade, foram excluídos. 


\section{INSTRUMENTOS}

Para a caracterização do perfil sociodemográfico e de saúde, foi utilizado um questionário semiestruturado, composto por informações referentes ao sexo, faixa etária, cor, estado conjugal, renda mensal em salário mínimo (SM), aposentadoria, percepção de saúde, motivo de ida a UBS, uso de medicamentos, histórico de quedas no último semestre e histórico de quase quedas no último semestre.

Para avaliação da funcionalidade nas ABVD foi utilizada a Escala de Katz (KATZ et al., 1963), que avalia a independência no desempenho de seis funções consideradas básicas: banhar-se, vestir-se, usar o banheiro, transferir-se, ter continência e alimentar-se. Cada item da Escala segue um escore de 0 a 3, representando o nível de dependência e independência do indivíduo da seguinte forma: 0) independência completa, 1) necessidade de ajuda não humana, 2) assistência humana, 3) completa dependência (LINO et al., 2008).

A Escala de Lawton avaliou o desempenho na realização das AIVD (LAWTON et al., 1969), e trata-se de uma medida genérica do nível de independência da pessoa idosa que avalia tarefas adaptativas ou necessárias para a vida independente na comunidade, tais como: usar o telefone, fazer compras, preparação da alimentação, lida da casa, lavagem da roupa, uso de transportes, preparação da medicação e gestão do dinheiro, mediante a atribuição de uma pontuação segundo a capacidade do sujeito avaliado para realizar essas atividades. A escala permite classificar os sujeitos em dependentes e independentes. A pontuação de máxima independência é sete e a maior dependência é zero (SANTOS; VIRTUOSO JÚNIOR, 2008).

O nível de atividade física dos idosos foi avaliado utilizando-se a versão curta do Questionário Internacional de Atividade Física (IPAQ) O mesmo é composto por sete questões abertas e suas informações permitem estimar o tempo despendido, por semana, em diferentes dimensões de atividade física (caminhadas e esforços físicos de intensidades moderada e vigorosa) e de inatividade física (posição sentada). 0 nível de atividade física é classificado em sedentário, irregularmente ativo, ativo ou muito ativo. 0 comportamento sedentário foi avaliado por meio do tempo médio sentado em um dia de semana, e em um dia de final de semana (MATSUDO et al., 2001).

\section{PROCEDIMENTOS DE COLETA DE DADOS}

Inicialmente foi realizado contato com as Assessoria de Formação e Capacitação Permanente dos Trabalhadores de Saúde (CECAPS) com o intuito de se obter autorização para a realização da coleta de dados. Em seguida, o projeto de pesquisa foi aprovado pelo Comitê de Ética em Pesquisa do Centro Universitário de Maringá (UNICESUMAR) por meio do parecer 1.626.966/2016, de acordo com as normas da Resolução 466/12 do Conselho Nacional de Saúde sobre pesquisa envolvendo seres humanos.

Os idosos voluntários foram abordados pelos pesquisadores, sendo informados quanto à justificativa, objetivos e procedimentos a serem realizados. Após essa abordagem, aqueles que aceitaram participar da pesquisa, assinaram o Termo de Consentimento Livre e Esclarecido (TCLE). A coleta foi realizada em dias, turnos e horários diversos, de acordo com a disponibilidade dos pesquisadores. Optou-se pela entrevista direta na aplicação dos questionários, em razão da possível dificuldade de leitura, problemas visuais e de compreensão dos questionamentos. 


\section{ANÁLISE DOS DADOS}

A análise foi realizada utilizando-se o Software SPSS 22.0, mediante uma abordagem de estatística descritiva e inferencial. Para as variáveis categóricas utilizou-se frequência e percentual; para as variáveis numéricas, inicialmente foi verificada a normalidade dos dados por meio do teste Kolmogorov-Smirnov. Como os dados não apresentaram distribuição normal, foram utilizadas mediana (Md) e quartis (Q1; Q3) para a caracterização dos resultados. Na estatística inferencial, empregou-se o teste do Qui-quadrado para tendência, no intuito de investigar a associação do nível de atividade física com as variáveis sociodemográficas, as condições de saúde e a capacidade funcional dos idosos. $\mathrm{Na}$ comparação da capacidade funcional em função das variáveis sociodemográficas e das condições de saúde, foi utilizado o teste de Kruskal-Wallis e o "U" de Mann-Whitney. A correlação entre o nível de atividade física e a capacidade funcional foi verificada por meio do coeficiente de Spearman. Considerou-se um nível de significância de $p<0,05$.

\section{RESULTADOS}

Dos 654 idosos avaliados, verificou-se o predomínio de idosos do sexo feminino $(56,0 \%)$, casados $(61,3 \%)$, com idade entre 60 e 69 anos $(59,2 \%)$, renda mensal de um a dois SM $(70,0 \%)$, da cor branca $(81,0 \%)$ e aposentados $(75,0 \%)$. Observou-se também que a maioria dos idosos possuem ensino fundamental incompleto $(43,0 \%)$.

Ao comparar a capacidade funcional dos idosos analisados em função das variáveis sociodemográficas, verificou-se diferença significativa nas ABVD e AIVD apenas para a faixa etária ( $p=0,001$ e $p=0,014$, respectivamente), indicando que os idosos na faixa etária de 60 a 69 anos apresentaram melhor capacidade funcional. Também foi encontrada diferença significativa nas AIVD em função da aposentadoria $(p=0,010)$, evidenciando que os idosos que ainda não são aposentados apresentaram melhor capacidade funcional.

Na comparação da capacidade funcional dos idosos em função das condições de saúde, verificou-se diferença significativa nas ABVD e AIVD para o motivo de ida a UBS ( $p=0,001$ e $p=0,014$, respectivamente), indicando que os idosos que procuram a UBS para visita médica apresentaram melhor capacidade funcional. Foi encontrada diferença significativa nas AIVD em função do nível de atividade física $(p=0,009)$, evidenciando que os idosos muito ativos/ativos fisicamente apresentaram melhor capacidade funcional. Também houve diferença significativa nas ABVD em função do histórico de quase quedas $(p=0,014)$, o que demonstra a melhor capacidade funcional para os idosos que não tinham histórico de quedas.

A Tabela 1 apresenta a associação do nível de atividade física com as variáveis sociodemográficas dos idosos. 
Tabela 1. Associação do nível de atividade física com as variáveis sociodemográficas dos idosos usuários das unidades básicas de saúde do município de Maringá, Paraná, 2016 (n=654).

\begin{tabular}{|c|c|c|c|c|c|}
\hline \multirow{3}{*}{ VARIÁVEIS } & \multicolumn{3}{|c|}{ Nível de atividade física } & \multirow{3}{*}{$\mathbf{X} 2$} & \multirow{3}{*}{$\mathbf{p}$} \\
\hline & $\begin{array}{c}\text { Muito ativo/ } \\
\text { ativo }\end{array}$ & Irreg. Ativo & Sedentário & & \\
\hline & (\%) & $(\%)$ & $(\%)$ & & \\
\hline \multicolumn{6}{|l|}{ Sexo } \\
\hline Masculino & $161(55,9)$ & $99(34,4)$ & $28(9,7)$ & \multirow{2}{*}{0,800} & \multirow{2}{*}{0,371} \\
\hline Feminino & $236(64,5)$ & $81(22,1)$ & $49(13,4)$ & & \\
\hline \multicolumn{6}{|l|}{ Faixa etária } \\
\hline 60 a 69 anos & $248(64,1)$ & $97(25,1)$ & $42(10,8)$ & \multirow{3}{*}{5,661} & \multirow{3}{*}{$0,017^{*}$} \\
\hline 71 a 79 anos & $117(59,4)$ & $55(27,9)$ & $25(12,7)$ & & \\
\hline 80 anos ou mais & $32(45,7)$ & $28(40,0)$ & $10(14,3)$ & & \\
\hline \multicolumn{6}{|l|}{ Estado conjugal } \\
\hline Casado & $241(60,1)$ & $115(28,7)$ & $45(11,2)$ & \multirow{5}{*}{0,046} & \multirow{5}{*}{0,831} \\
\hline Solteiro & $32(55,2)$ & $18(31,0)$ & $8(13,8)$ & & \\
\hline Divorciado/Separado & $49(66,2)$ & $17(23,0)$ & $8(10,8)$ & & \\
\hline Viúvo & $75(62,0)$ & $30(24,8)$ & $16(13,2)$ & & \\
\hline Cor & & & & & \\
\hline Branca & $318(60,0)$ & $144(27,2)$ & $68(12,8)$ & \multirow{3}{*}{1,989} & \multirow{3}{*}{0,158} \\
\hline Preta & $65(61,9)$ & $33(31,4)$ & $7(6,7)$ & & \\
\hline Outras & $14(73,7)$ & $3(15,8)$ & $2(10,5)$ & & \\
\hline \multicolumn{6}{|l|}{ Escolaridade } \\
\hline Analfabeto & $42(53,2)$ & $27(34,2)$ & $10(12,7)$ & \multirow{6}{*}{4,473} & \multirow{6}{*}{$0,034^{*}$} \\
\hline Fund. Incompleto & $161(57,3)$ & $88(31,3)$ & $32(11,4)$ & & \\
\hline Fund. Completo & $92(62,6)$ & $36(24,5)$ & $19(12,9)$ & & \\
\hline Médio completo & $65(67,7)$ & $21(21,9)$ & $10(10,4)$ & & \\
\hline Superior & $37(72,5)$ & $8(15,7)$ & $6(11,8)$ & & \\
\hline Aposentadoria & & & & & \\
\hline
\end{tabular}




\begin{tabular}{|c|c|c|c|c|c|}
\hline \multirow{2}{*}{ VARIÁVEIS } & \multicolumn{3}{|c|}{ Nível de atividade física } & \multirow{2}{*}{ X } & \multirow{2}{*}{ p } \\
\cline { 2 - 5 } & $\begin{array}{c}\text { Muito ativo/ } \\
\text { ativo }\end{array}$ & Irreg. Ativo & Sedentário & X2 & \\
\cline { 2 - 5 } & $\mathbf{( \% )}$ & $\mathbf{( \% )}$ & $\mathbf{( \% )}$ & & \\
\hline Sim & $289(59,5)$ & $138(28,5)$ & $58(12,0)$ & \multirow{2}{*}{0,745} & 0,388 \\
Não & $104(64,2)$ & $40(24,7)$ & $18(11,1)$ & & \\
1 a 2 SM & \multicolumn{2}{|c|}{ Renda mensala } & & \\
2,1 a 3 SM & $272(62,8)$ & $107(24,7)$ & $54(12,5)$ & & \\
Mais de 3 SM & $84(53,8)$ & $54(34,6)$ & $18(11,5)$ & 0,455 & 0,500 \\
\hline
\end{tabular}

*Associação significativa - $p<0,05$ : Teste de Qui-quadrado para proporções.

Fund: Fundamental; SM: Salário (s) mínimo (s); Irreg.: irregularmente.

Verificou-se (Tabela 1) associação significativa do nível de atividade física apenas com a faixa etária $(p=0,017)$ e o nível de escolaridade $(p=0,034)$. Este achado indica a maior prevalência de idosos com nível de atividade física muito ativo/ativo na faixa etária de 60 a 69 anos ( $f=248)$ e com ensino fundamental incompleto $(\mathbf{f}=161)$.

A Tabela 2 apresenta a associação do nível de atividade física com as condições de saúde e capacidade funcional dos idosos.

Tabela 2. Associação do nível de atividade física com as condições de saúde e capacidade funcional dos idosos usuários das unidades básicas de saúde do município de Maringá, Paraná, 2016 $(n=654)$.

\begin{tabular}{|c|c|c|c|c|c|}
\hline \multirow{3}{*}{ VARIÁVEIS } & \multicolumn{3}{|c|}{ Nivel de atividade física } & \multirow{3}{*}{$\mathbf{X}^{2}$} & \multirow{3}{*}{$\mathbf{p}$} \\
\hline & $\begin{array}{l}\text { Muito ati- } \\
\text { vo/ ativo }\end{array}$ & Irreg. Ativo & Sedentário & & \\
\hline & $(\%)$ & $(\%)$ & $(\%)$ & & \\
\hline \multicolumn{6}{|l|}{ Percepção de saúde } \\
\hline Boa & $196(62,0)$ & $89(28,2)$ & $31(9,8)$ & & \\
\hline Regular & $167(63,7)$ & $66(25,2)$ & $29(11,1)$ & 5,882 & $0,015 *$ \\
\hline Ruim & $33(44,6)$ & $25(33,8)$ & $16(21,6)$ & & \\
\hline Motivo da ida a UBS & & & & & \\
\hline
\end{tabular}




\begin{tabular}{|c|c|c|c|c|c|}
\hline \multirow{3}{*}{ VARIÁVEIS } & \multicolumn{3}{|c|}{ Nível de atividade física } & \multirow{3}{*}{$\mathbf{X}^{2}$} & \multirow{3}{*}{$\mathbf{p}$} \\
\hline & \multirow{2}{*}{\begin{tabular}{|c|}
$\begin{array}{c}\text { Muito ati- } \\
\text { vo/ ativo }\end{array}$ \\
$(\%)$ \\
\end{tabular}} & \multirow{2}{*}{$\begin{array}{c}\text { Irreg. Ativo } \\
(\%) \\
\end{array}$} & \multirow{2}{*}{$\begin{array}{c}\text { Sedentário } \\
(\%) \\
\end{array}$} & & \\
\hline & & & & & \\
\hline Visita médica & $175(60,1)$ & $76(26,1)$ & $40(13,7)$ & & \\
\hline Buscar medicamentos & $122(57,0)$ & $68(31,8)$ & $24(11,2)$ & 2,336 & 0,126 \\
\hline Realização de exames & $98(67,6)$ & $34(23,4)$ & $13(9,0)$ & & \\
\hline \multicolumn{6}{|l|}{ Medicamentos } \\
\hline Nenhum & $63(65,6)$ & $25(26,0)$ & $8(8,3)$ & \multirow{3}{*}{1,350} & \multirow{3}{*}{0,245} \\
\hline 1 a 2 & $164(57,5)$ & $93(32,6)$ & $28(9,8)$ & & \\
\hline Mais de 2 & $165(61,6)$ & $62(23,1)$ & $41(15,3)$ & & \\
\hline \multicolumn{6}{|l|}{ Quedas } \\
\hline Sim & $60(56,6)$ & $31(29,2)$ & $15(14,2)$ & \multirow{2}{*}{1,090} & \multirow{2}{*}{0,297} \\
\hline Não & $336(61,5)$ & $148(27,1)$ & $62(11,4)$ & & \\
\hline \multicolumn{6}{|l|}{ Quase quedas } \\
\hline Sim & $80(60,6)$ & $40(30,3)$ & $12(9,1)$ & \multirow{2}{*}{0,242} & \multirow{2}{*}{0,623} \\
\hline Não & $315(60,7)$ & $139(26,8)$ & $65(12,5)$ & & \\
\hline \multicolumn{6}{|l|}{ ABVD } \\
\hline Independente & $390(61,4)$ & $172(27,1)$ & $73(11,5)$ & \multirow{3}{*}{4,421} & \multirow{3}{*}{$0,035^{*}$} \\
\hline Dependente parcial/total & $7(36,8)$ & $8(42,1)$ & $4(21,1)$ & & \\
\hline \multicolumn{4}{|l|}{ AIVD } & & \\
\hline Independente & $329(63,1)$ & $140(26,9)$ & $52(10,0)$ & \multirow{2}{*}{9,463} & \multirow{2}{*}{$0,002^{*}$} \\
\hline Dependente parcial/total & $68(51,1)$ & $40(30,1)$ & $25(18,8)$ & & \\
\hline
\end{tabular}

*Associação significativa - $p<0,05$ : Teste de Qui-quadrado para proporções.

Irreg: irregularmente; ABVD: atividades básicas de vida diária; AIVD: atividades Instrumentais de vida diária.

Foi encontrada associação significativa do nível de atividade física apenas com a percepção de saúde $(p=0,015)$, ABVD $(p=0,035)$ e AIVD $(p=0,002)$, evidenciando que parece existir uma associação e tendência dos idosos com boa percepção de saúde e independência nas ABVD e AIVD terem nível de atividade física muito ativo/ativo (Tabela 2). As demais condições de saúde não se associaram significativamente $(p>0,05)$ com o nível de atividade física (Tabela 2). 
Ao analisar a correlação entre o nível de atividade física e a capacidade funcional dos idosos (Tabela 3), verificou-se que as ABVD apresentaram correlação significativa $(p<0,05)$, positiva e fraca $(r<0,40)$ com todas as variáveis relacionadas à prática de atividades físicas leves e moderadas. Já as AIVD apresentaram correlação significativa $(p<0,05)$, fraca e positiva com as variáveis relacionadas à prática de atividades físicas leves, e negativa com as variáveis relacionadas à prática de atividades físicas vigorosas. Tais achados parecem indicar que a realização de atividades leves e moderadas está associada a melhor capacidade funcional, enquanto as atividades vigorosas estão associadas com a maior dependência nas AIVD.

Tabela 3. Correlação entre o nível de atividade física e a capacidade funcional dos idosos. Maringá, Paraná, 2016 (n=654).

\begin{tabular}{|c|c|c|c|c|c|c|c|c|c|c|c|}
\hline \multirow{2}{*}{ VARIÁVEIS } & \multicolumn{9}{|c|}{ Nivel de atividade física } & \multirow{2}{*}{$\begin{array}{c}\text { ABVD } \\
10 \\
\end{array}$} & \multirow{2}{*}{$\begin{array}{c}\text { AIVD } \\
11\end{array}$} \\
\hline & 1 & 2 & 3 & 4 & 5 & 6 & 7 & 8 & 9 & & \\
\hline 1. & & $0,56^{*}$ & $0,77^{*}$ & $0,23^{*}$ & $0,14^{*}$ & $0,18^{*}$ & $-0,06$ & $-0,08$ & $-0,07$ & 0,10 * & $0,21^{*}$ \\
\hline 2. & & & $0,89 *$ & $0,26^{*}$ & $0,31^{*}$ & $0,28^{*}$ & 0,02 & 0,01 & 0,01 & $0,10^{*}$ & $0,14^{*}$ \\
\hline 3. & & & & $0,26^{*}$ & $0,26^{*}$ & 0,30 * & 0,02 & 0,01 & 0,01 & $0,11^{*}$ & $0,16^{*}$ \\
\hline 4. & & & & & $0,86^{*}$ & $0,93^{*}$ & $0,47^{*}$ & $0,47^{*}$ & $0,47^{*}$ & $0,10^{*}$ & $-0,01$ \\
\hline 5. & & & & & & $0,94^{*}$ & 0,50 * & $0,53^{*}$ & $0,53^{*}$ & 0,10 * & 0,03 \\
\hline 6. & & & & & & & $0,51^{*}$ & $0,53^{*}$ & $0,53^{*}$ & $0,10^{*}$ & 0,02 \\
\hline 7. & & & & & & & & $0,98^{*}$ & $0,98^{*}$ & 0,05 & $-0,12^{*}$ \\
\hline 8. & & & & & & & & & $0,99^{*}$ & 0,05 & $-0,10^{*}$ \\
\hline 9. & & & & & & & & & & 0,05 & $-0,10^{*}$ \\
\hline 10. & & & & & & & & & & & $0,35^{*}$ \\
\hline 11. & & & & & & & & & & & \\
\hline
\end{tabular}

*Correlação Significativa $-p<0,05$. Nota: 1. Dias de caminhada; 2. Min. caminhada p/ dia; 3. Min. caminhada p/ sem.; 4. Dias atv moderada; 5. Min. Ativ. Mod. p/ dia; 6. Min. Ativ. Mod. p/ sem; 7. Dias de atv. Vigorosa; 8. Min. Atv. vigorosa p/ dia; 9. Min. Atv. Vig. p/ sem.; 10. ABVD; 11. AIVD.

Min.: minutos; Atv.: atividades; Mod.: moderada; p/sem.: por semana; ABVD: atividade básica de vida diária; $A I V D=$ atividade Instrumental de vida diária

\section{DISCUSSÃO}

Os principais achados deste estudo são: idosos na faixa etária de 60 a 69 anos, não aposentados, que procuram as UBS para consulta médica, que são mais ativos fisicamente, e que não possuem histórico 
de queda no último semestre, apresentaram melhor capacidade funcional. Há maior prevalência de idosos com nível de atividade física muito ativo/ativo na faixa etária de 60 a 69 anos e com ensino fundamental incompleto; e que parece existir uma associação e tendência dos idosos com boa percepção de saúde e independência nas ABVD e AIVD terem nível de atividade física muito ativo/ativo

Os idosos entrevistados apresentaram ser na maioria independentes nas ABVD e AIVD. Barbosa et al. (2014) inferem que com o aumento da expectativa de vida e com o avanço de estudos que buscam promover a qualidade de vida das pessoas idosas, o número de idosos mais ativos e independentes é cada vez maior.

Verificou-se comprometimento funcional mais evidente para realização das AIVD, atividades mais elaboradas e de maior complexidade, como também observado em outros estudos nacionais (FREITAS et al., 2012; VIRTUOSO JÚNIOR et al., 2015) e internacionais (GUREJE et al., 2006; ROCHAT et al., 2010), independentemente dos procedimentos adotados na sua mensuração. Hierarquicamente, as perdas ocorrem de AIVD para ABVD devido às AIVD serem consideradas tarefas mais adaptativas para a vida independente na comunidade e exigirem maior integridade física e cognitiva (FREITAS et al., 2012; VIRTUOSO JÚNIOR et al., 2015; MILLÁN-CALENTI et al., 2010) e, portanto, de execução mais difícil e complexa do que as atividades básicas. Essa progressão da incapacidade tem implicações importantes para a saúde pública, uma vez que o (re)conhecimento e a prevenção dos fatores de risco, bem como o cuidado em caso de detecção de alguma incapacidade para a AIVD, representam etapas essenciais à prevenção de futura limitação na realização das ABVD e às potenciais consequências ao indivíduo, ao cuidador, às famílias e ao sistema de saúde (FIALHO et al., 2014).

Todavia, na literatura verificam-se divergências na frequência de dependência tanto em ABVD quanto em AIVD (VIRTUOSO JÚNIOR et al., 2015; MILLÁN-CALENTI et al., 2010), em relação ao presente estudo, que foi de 2,9\% e 20,3\% respectivamente. Possivelmente essas discrepâncias se devem às diferentes realidades socioeconômicas e culturais e aos processos de mensuração utilizados.

De modo geral, os idosos participantes do estudo apresentaram características sociodemográficas semelhantes a outros estudos com idosos brasileiros na atenção primária. Esse fato reforça o potencial de generalização dos resultados aqui apresentados.

0 presente estudo apontou melhor capacidade funcional nas ABVD e AIVD em idosos na faixa etária de 60 a 69 anos, e que procuram as UBS para consulta médica; e nas ABVD para os idosos sem histórico de quedas, e nas AIVD para os idosos que ainda não estavam aposentados e os idosos muito ativos/ativos fisicamente.

Com o avanço da idade, verifica-se maior probabilidade de dependência tanto nas AIVD quanto nas ABVD, uma vez que a capacidade de realizar uma tarefa envolve a integração de múltiplos sistemas que com o passar dos anos, gradualmente entram em declínio (FIALHO et al., 2014). Os idosos apresentam maior dependência funcional em comparação aos adultos e jovens e, no grupo idoso, indivíduos mais velhos tendem a ser menos independentes que os mais jovens.

A ocorrência de quedas em pessoas idosas é de cerca de $30 \%$, tem como consequências, além de possíveis fraturas e risco de morte, o medo de cair, a restrição de atividades físicas, o declínio na saúde e o aumento do risco de institucionalização e podem representar uma perda significativa da 
capacidade funcional (PEREIRA et al., 2012). 0 relato de quedas nos últimos três meses mostrou-se com um indicador de pior capacidade funcional no estudo de base populacional com amostra de 397 idosos residentes na zona urbana da cidade de Ubá/MG (TSUBOTA-UTSUGI et al., 2011). Além disso, um idoso que sofreu uma queda pode desenvolver o que se chama de "síndrome pós-queda", em que o impacto psicológico pode levar à diminuição das ABVD (NUNES et al., 2009).

Muitos dos fatores de risco para quedas contribuem também para imobilidade e declínio funcional. Idosos sedentários têm mais de 15 vezes mais chances de quedas do que os ativos (FABRíCIO et al., 2004). É importante destacar que normalmente idosos sedentários apresentam diminuição da capacidade funcional e esse déficit influencia no aumento da incidência de quedas. Esta, por sua vez, tem relação direta com prejuízo do equilíbrio, comprometimento das atividades instrumentais, limitações da mobilidade articular, da marcha e da força muscular (PERRACINI; RAMOS, 2002). A prática de atividade física pode ter efeito positivo sobre a estabilidade postural e sobre o risco de quedas, proporcionando aumento do equilíbrio e da coordenação, da mobilidade e força e da capacidade funcional (SKELTON, 2001).

Aliado à prática de atividade física, manter-se ativo economicamente, seja por meio de aposentadorias e pensões, ou por realização de trabalhos remunerados, contribui para a independência funcional (RIBEIRO et al., 2015). Os autores verificaram que os idosos que mantiveram uma ocupação possuíram maiores médias na Medida de Independência Funcional (MIF) comparado aos que não estão mais no mercado de trabalho e tiveram altos escores em atividades como resolução de problemas e interação social, capacidades necessárias para a realização de tarefas como gerir o próprio dinheiro e fazer compras.

Os idosos brasileiros têm-se revelado os principais provedores da renda familiar, situação que thes confere maior valorização dentro da família e da sociedade em geral. 0 aumento das taxas de chefia entre idosos e a redução na proporção que reside na casa de parentes sugerem uma diminuição na dependência dos idosos. Além disso, a diminuição da renda pode estar relacionada à dificuldade de aquisição de medicamentos, a procura por serviços de saúde, como também aumenta a dificuldade em boa alimentação contribuindo para a perda ou diminuição da capacidade funcional (NERI; SOARES, 2007).

Em relação ao nível de atividade física muito ativo/ativo foi encontrada associação significativa com a faixa etária de 60 a 69 anos, nível de escolaridade do ensino fundamental incompleto, boa percepção de saúde e independência nas ABVD e AIVD.

A idade pode ser um fator limitante para a prática de atividade física, pois com o avançar dos anos, as condições fisiológicas impedem a realização de diversos exercícios que contribuem para uma vida mais saudável (CASSOU et al., 2008). Já a relação entre atividade física e escolaridade aponta para uma realidade bem comum aos idosos no Brasil. A necessidade do ingresso no mercado de trabalho informal, para a manutenção das condições básicas de vida, faz com que esses sujeitos sejam incentivados a abandonar o processo de escolarização na mais tenra idade e dar início a uma jornada de trabalho dissociada dos estudos, da formação profissional e dos cuidados com a saúde. Culturalmente, a lógica da evasão escolar está instalada no país e diluída como um projeto político de 'emburrecimento', não obstante o aumento da permanência desses idosos do 
mercado de trabalho, faz com que só com a advento da aposentadoria, eles possam retornar ao protagonismo de suas vidas e estarem mais atentos as questões de funcionalidade na velhice (MELO et al., 2009).

A auto percepção de saúde é um importante indicador da saúde dos idosos e tem sido amplamente utilizada em pesquisas, uma vez que se mostra como uma medida da atual situação de saúde e bem-estar dos indivíduos (BORIM et al., 2012). Além disso, a auto percepção de saúde tem se mostrado preditora de morbimortalidade e incapacidade especialmente em pessoas idosas (BLAZER, 2008; BORIM et al., 2012).

O nível de atividade física é relatado como fundamental para manutenção da autonomia, independência, capacidade funcional, além do retardo ou reversão de processos patológicos em andamento, podendo ainda apresentar benefícios psicológicos como melhora da autoestima, criatividade e envelhecimento saudável e ativo (FERREIRA et al., 2012).

Destaca-se como limitação deste estudo o delineamento transversal que limita a avalição da causa e efeito das variáveis, devendo, portanto, a interpretação dos resultados ser cuidadosa. Ainda, a utilização de questionários para mensurar o nível de atividade física também é uma limitação do estudo, uma vez que é uma medida subjetiva e pode mascarar os resultados, entretanto, foi utilizado neste estudo o questionário (IPAQ) mais reconhecido na literatura. Com isso, futuras pesquisas devem avaliar as variáveis deste estudo por meio de um desenho longitudinal e utilizando medidas mais confiáveis, como o acelerômetro. Sugere-se ainda novos estudos com idosos usuários da atenção básica a saúde, relacionando a atividade física e a funcionalidade com diversas variáveis, sejam elas socioeconômicas, de saúde ou de outra natureza, afim de se conhecer melhor os fatores que se associam as mesmas.

\section{CONCLUSÃO}

A partir dos resultados encontrados, pode-se concluir que as variáveis sociodemográficas e as condições de saúde estão associadas ao nível de atividade física e a capacidade funcional de idosos. Além disso, destaca-se que a prática de atividades físicas leves e moderadas podem favorecer a meIhor capacidade funcional, enquanto a prática de atividades físicas vigorosas parece estar inversamente associada à capacidade funcional.

A avaliação da capacidade funcional representa um instrumento fundamental para a assistência aos idosos, uma vez que pode nortear a prevenção, o tratamento mais adequado das diferentes doenças ou agravos, a recuperação funcional e a definição de estratégias para a saúde do idoso.

Os resultados encontrados nesta pesquisa são importantes para nortear ações de profissionais atuantes em Unidades de Saúde da Família e nos Núcleos de Apoio à Saúde da Família, no sentido de incentivar a prática de atividade física, prevenir quedas, identificar indivíduos em situação de risco para queda, e promover a capacidade funcional dos idosos. 


\section{REFERÊNCIA}

ALVES, J.E.D. Transição demográfica, transição da estrutura etária e envelhecimento. Rev

Longeviver, v. 40, n. 4, p. 8-15, 2014.

BARBOSA, B.R. et al. Avaliação da capacidade funcional dos idosos e fatores associados à incapacidade. Ciên Saúde Col, v. 19, n. 8, p. 3317-3325, 2014.

BERTOLI, J. et al. Six weeks of Mat Pilates training are enough to improve functional capacity in elderly women. J Bodyw Mov Ther, v. 21, n. 4, p. 1003-1008, 2017.

BLAZER, D.G. How do you feel about? Health outcomes in late life and self-perceptions of health and well-being. Gerontologist, v. 48, n. 4, p. 415-422, 2008.

BORIM, F.S.A. Autoavaliação da saúde em idosos: pesquisa de base populacional no Município de Campinas, São Paulo, Brasil. Cad Saúde Públ, v. 28, p. 769-780, 2012.

BRUCKI, S.M.D. et al. Sugestões para o uso do mini-exame do estado mental no Brasil. Arq Neuropsiquiat, v. 61, n. 3B, p. 777-81, 2003.

CASSOU, A. C. et al. Barreiras para a atividade física em idosos: uma análise por grupos focais. J Phys Educ, v. 19, n. 3, p. 353-360, 2008.

CORDEIRO, J. et al. Efeitos da atividade física na memória declarativa, capacidade funcional e qualidade de vida em idosos. Rev Bras Geriat Gerontol, v. 17, n. 3, p. 541-552, 2014.

CRUZ-JENTOFT, A. J. European Working Group on Sarcopenia in Older People: Sarcopenia: European consensus on definition and diagnosis. Report of the European Workign Group on Sarcopenia in Older People. Age Ageing, v. 39, n. 1, p. 412-423, 2010.

FABRÍCIO, S.C.C. et al. Causas e conseqüências de quedas de idosos atendidos em hospital público. Rev Saúde Públ, v. 38, n. 1, p. 93-99, 2004.

FARIAS, N.; BUCHALLA, C. M. A classificação internacional de funcionalidade, incapacidade e saúde da organização mundial da saúde: conceitos, usos e perspectivas. Rev Bras Epidemiol, v. 8, p. 187193, 2005.

FERREIRA, O. G. L. et al. Envejecimiento activo y su relación con la independencia funcional. Texto Contexto-Enferm, v. 21, n. 3, p. 513-518, 2012. 
FIALHO, C. B. et al. Capacidade funcional e uso de serviços de saúde por idosos da Região Metropolitana de Belo Horizonte, Minas Gerais, Brasil: um estudo de base populacional. Cad Saúde Públ, v. 30, n. 3, p. 599-610, 2014.

FOLSTEIN, M. F. et al. The mini-mental state examination. Arch Gen Psych, v. 40, n. 7, p. 812-812, 1983.

FREITAS, R. S. et al. Capacidade funcional e fatores associados em idosos: estudo populacional. Acta Paul Enferm, v. 25, n. 6, p. 933-939, 2012.

GUREJE, O. et al. Functional disability in elderly Nigerians: results from the Ibadan Study of Aging. J Am Geriatr Soc, v. 54, n. 11, p. 1784-1789, 2006.

HALLAL, P. C. et al. Global physical activity levels: surveillance progress, pitfalls, and prospects. Lancet, v. 380, n. 9838, p. 247-257, 2012.

JACINTO, J. L. et al. Efeitos da caminhada em ritmo prescrito e autosselecionado sobre a capacidade funcional de mulheres idosas. J Health Sci, v. 18, n. 4, p. 257-263, 2017.

KATZ, S. et al. Studies of illness in the aged. The index of old: A standardized measure of biological and psychosocial function. JAMA, v. 185, n. 1, p. 914-919, 1963.

KNUTH, A. G. et al. Changes in physical activity among Brazilian adults over a 5-year period. J Epidemiol Commun Health, v. 64, n. 7, p. 591-595, 2010.

LAWTON, M. P. et al., Utilisateurs. Instrumental activities of daily living (IADL). Gerontologist, v. 9, p. 179-186, 1969.

LINO, V. T. S. et al. Cross-cultural adaptation of the independence in activities of daily living index (Katz index). Cad Saude Públ, v. 24, n. 1, p. 103-112, 2008.

LOPES, G. L.; SANTOS, M. I. P. O. Funcionalidade de idosos cadastrados em uma unidade da Estratégia Saúde da Família segundo categorias da Classificação Internacional de Funcionalidade. Rev Bras Geriatr Gerontol, v. 18, n. 1, p. 71-83, 2015.

MALTA, D. et al. Política Nacional de Promoção da Saúde, descrição da implementação do eixo atividade física e práticas corporais, 2006 a 2014. Rev Bras Ativ Física Saúde, v. 19, n. 3, p. 286-286, 2014.

MATSUDO, S. et al. Questinário internacional de atividade física (IPAQ): estudo de validade e reprodutibilidade no Brasil. Rev Bras Ativ Física Saúde, v. 6, n. 2, p. 05-18, 2001. 
MELO, M. C. et al. A educação em saúde como agente promotor de qualidade de vida para o idoso. Ciên Saúde Col, v. 14, p. 1579-1586, 2009.

MILLÁN-CALENTI, J. C. et al. Prevalence of functional disability in activities of daily living (ADL), instrumental activities of daily living (IADL) and associated factors, as predictors of morbidity and mortality. Arch Gerontol Geriatr, v. 50, n. 3, p. 306-310, 2010.

NERI, M.C.; SOARES, W. L. Estimando o impacto da renda na saúde através de programas de transferência de renda aos idosos de baixa renda no Brasil. Cad Saúde Públ, v. 23, p. 1845-1856, 2007.

NUNES, M. C. R. et al. Influência das características sociodemográficas e epidemiológicas na capacidade funcional de idosos residentes em Ubá, Minas Gerais. Braz J Physic Ther, v. 13, n. 5, p.376-382, 2009.

PAULA, A. F. M. et al. Functionality profiles related to mortality in elders assisted in a Geriatric Outpatient Service. Cad Bras Ter Ocup, v. 23, n. 1, p. 153-162, 2015.

PEREIRA, G. N. et al. Indicadores demográficos e socioeconômicos associados à incapacidade funcional em idosos. Cad Saúde Públ, v. 28, n. 11, p. 2035-2042, 2012.

PERRACINI, M. R.; RAMOS, L. R. Fatores associados a quedas em uma coorte de idosos residentes na comunidade. Rev Saúde Públ, v. 36, p. 709-716, 2002.

RIBEIRO, D. K. M. N. et al. Fatores contributivos para a independência funcional de idosos longevos. Rev Esc Enferm USP, v. 49, n. 1, p. 89-96, 2015.

ROCHAT, S. et al. Frailty and use of health and community services by community-dwelling older men: the Concord Health and Ageing in Men Project. Age Ageing, v. 39, n. 2, p. 228-233, 2010.

SANTOS, R. L.; VIRTUOSO JÚNIOR, J. S. Confiabilidade da versão brasileira da escala de atividades instrumentais da vida diária. Rev Bras Prom Saúde, v. 21, n. 4, p. 290-296, 2008.

SIQUEIRA, F. C. V. et al. Atividade física em profissionais de saúde do Sul e Nordeste do Brasil. Cad Saúde Públ, v. 25, p. 1917-1928, 2009.

SKELTON, D. A. Effects of physical activity on postural stability. Age Ageing, v. 30, n. 4, p. 33-39, 2001.

SPOSITO, G. et al. Atividades avançadas de vida diária (AAVDs) e o desempenho cognitivo em idosos residentes na comunidade: Dados do Estudo FIBRA Polo UNICAMP. Rev Bras Geriatr Gerontol, v. 19, n. 1, p. 7-20, 2016. 
TSUBOTAUTSUGI, M. et al. Health Behaviors as Predictors for Declines in HigherLevel Functional Capacity in Older Adults: The O hasama Study. J Am Geriatr Soc, v. 59, n. 11, p. 1993-2000, 2011.

VIRTUOSO JÚNIOR, J. S. et al. Prevalence of disability and associated factors in the elderly. Texto Contexto-Enfermagem, v. 24, n. 2, p. 521529, 2015.
Recebido em: 30 de Março de 2019

Avaliado em: 16 de Julho de 2019

Aceito em: 16 de Julho de 2019

1 Fisioterapeuta e Profissional de Educação Física. Mestre em Promoção da Saúde e Doutor em Gerontologia, Centro Universitário Metropolitano de Maringá, Maringá, PR.

E-mail: d.vicentini@hotmail.com

2 Profissional de Educação Física. Centro Universitário Metropolitano de Maringá, Maringá, PR. E-mail: nathan_tripexx@hotmail.com

3 Fisioterapeuta. Mestre em Saúde Coletiva e Doutora em Ciências da Saúde, Universidade Estadual de Londrina, Londrina, PR. E-mail: celita@uel.br

4 Profissional de Educação Física. Mestre e Doutor em Educação Física, Universidade Federal do Vale do São Francisco, Petrolina, PE. E-mail: jroberto.jrs01@gmail.com

5 Pedagogo. Mestre em Eduação e Doutorando em Gerontologia, Universidade Estadual de Campinas, Campinas, SP. E-mail: danielpaguiar@hotmail.com

6 Fisioterapeuta. Mestre em Promoção da Saúde. Doutorando em Ciências da Reabilitação, Universidade de São Paulo, São Paulo, SP. E-mail: mateusantunes@usp.br

7 Fisioterapeuta. Mestre e Doutora em Ciências Morfofuncionais, Programa de Pós-Graduação em Promoção da Saúde, Centro Universitário de Maringá, PR. E-mail: smmgbertolini@yahoo.com.br

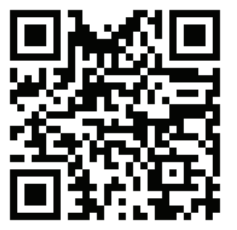

A autenticidade desse artigo pode ser conferida no site https://periodicos. set.edu.br
Este artigo é licenciado na modalidade acesso abertosob a Atribuição-Compartilhalgual CC BY-SA
(1)

GRUPO TIRADENTES Inspirando ofuturo

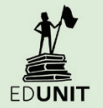


